\title{
Surface track potential created by fast protons at LiF surfaces
}

\author{
A. Arnau, ${ }^{1}$ M. S. Gravielle, ${ }^{2}$ J. E. Miraglia, ${ }^{2}$ and V. H. Ponce ${ }^{3}$ \\ ${ }^{1}$ Departamento de Fisica de Materiales, Facultad de Quimica and Centro Mixto CSIC-UPV/EHU, San Sebastian, Spain \\ ${ }^{2}$ Instituto de Astronomia y Fisica del Espacio, Consejo Nacional de Investigaciones Cientificas y Tecnicas, Departamento de Fisica, \\ Facultad de Ciencias Exactas y Naturales, Universidad de Buenos Aires, Buenos Aires, Argentina \\ ${ }^{3}$ Centro Atomico Bariloche, San Carlos de Bariloche, San Carlos, Argentina \\ and Donostia International Physics Center, DIPC, San Sebastian, Spain
}

(Received 26 December 2002; published 13 June 2003)

\begin{abstract}
A model based on atomic ionization of $\mathrm{F}^{-}$sites at the surface is used to calculate the surface track potential created by fast protons at $\mathrm{LiF}$ surfaces. For $100-\mathrm{keV}$ protons at grazing incidence, we find that the track potential close to the surface plays a significant role in determining the shift to lower energies of the convoy electron peak with respect to the unperturbed cusp value.
\end{abstract}

DOI: 10.1103/PhysRevA.67.062902

PACS number(s): 34.50.Dy, 34.50.Fa, 79.20.Rf, 78.90.+t

\section{INTRODUCTION}

The interaction of swift ions with solids has attracted the attention of physicist for more than a hundred years. However, there are still open questions that remain to be solved, which have not only fundamental interest but are also interesting in material science applications. One of them is the characterization of the so-called track potential, i.e., the potential that is created by the ionization of target atoms. In the case of metallic targets electrons are very efficient in filling the vacancies due to their high mobility. However, in the case of insulators the neutralization of the charge imbalance produced by ionization, i.e., the source of the track potential, may well last for a period of time long compared to the time scale set up by the moving ion. Therefore, one expects that the track potential plays a role in processes such as electron emission, energy loss, and charge exchange. Some theoretical work that considers effects due to the track potential in the interaction of multicharged ions with insulating surfaces $[1,2]$ or negative ion formation [3] has been done. The effects due to the track potential could be even more pronounced for collisions of ions on surfaces under small incidence angle because screening and neutralization are less efficient compared to the bulk case [4]. Recent experimental work have confirmed the importance of the track potential in: (i) shifts in the Auger line positions of electrons emitted after transmission of ions through thin foils [5], (ii) shift of the convoy electron peak positions in both transmission [6], and grazing incidence [7], (iii) acceleration of desorbed positive hydrogen ions [8], and (iv) a reduction in the energy loss and electron emission of fast ions scattered off $\mathrm{KCl}$ surfaces under highly grazing incidence [9].

In this work we present a model for the surface track potential. We consider fast protons interacting with $\mathrm{LiF}(100)$ surfaces. The surface is represented by an array of $\mathrm{F}^{-}$ions that are ionized by the passage of the proton. The source of the track potential are positive charges created at the crystal surface (lack of electrons). The distance dependence of the ionization probability determines the surface track potential by integration along the ion trajectory.

We evaluate the ionization probability from $\mathrm{F}^{-}$by employing a semiclassical model in which the multiple colli- sions of the incident ion with the surface ions are treated as single encounters with outermost target ions along the projectile path [10]. The ion trajectory is determined using a Moliere planar potential [11]. The ionization probability per unit path length is expressed in terms of atomic probabilities, which are evaluated with the continuum-distorted-waveeikonal-initial-state (CDW-EIS) approximation. This theory is a distorted-wave method that makes use of the eikonal wave function in the initial channel and the CDW wave function in the final channel. The CDW-EIS approximation has been found to be successful to explain the ionization process for a large variety of collision systems [12]. It takes into account the long-range behavior of the Coulomb potential, including the distortion produced by the projectile in both the initial and final channels. From the ionization probability we derive the track potential, which is produced by the target ionization along the ion path. We consider that during the characteristic collisional time (a few femtoseconds) all emitted electrons escape from the surface, either to vacuum or deep inside the crystal, and the microscopic charging up of the surface is not compensated.

In order to test the validity of our theory we calculate the ionization cross section and stopping cross section, and these are compared with the experimental ones. We also calculate the stopping power or the ionization probability per unit path length to illustrate some aspects of the description. The values of the track potential and the corresponding electric field that we obtain are used to justify the shift of the convoy electron peak to lower energy. Our qualitative explanation is based on a simple model that considers the reduction of the ionization threshold due to the perturbing field (approximately constant around the projectile). Other aspects related to the track potential are discussed as well.

\section{THEORY}

\section{A. Surface track potential}

We consider a heavy projectile $P$ of charge $q$ and mass $M_{P}$ impinging grazingly on a solid surface. As a result of the collision, an electron $e$ that is initially bound to a target ion in the state $i$ with energy $\varepsilon_{i}$, is emitted with momentum $\vec{k}_{f}$, 


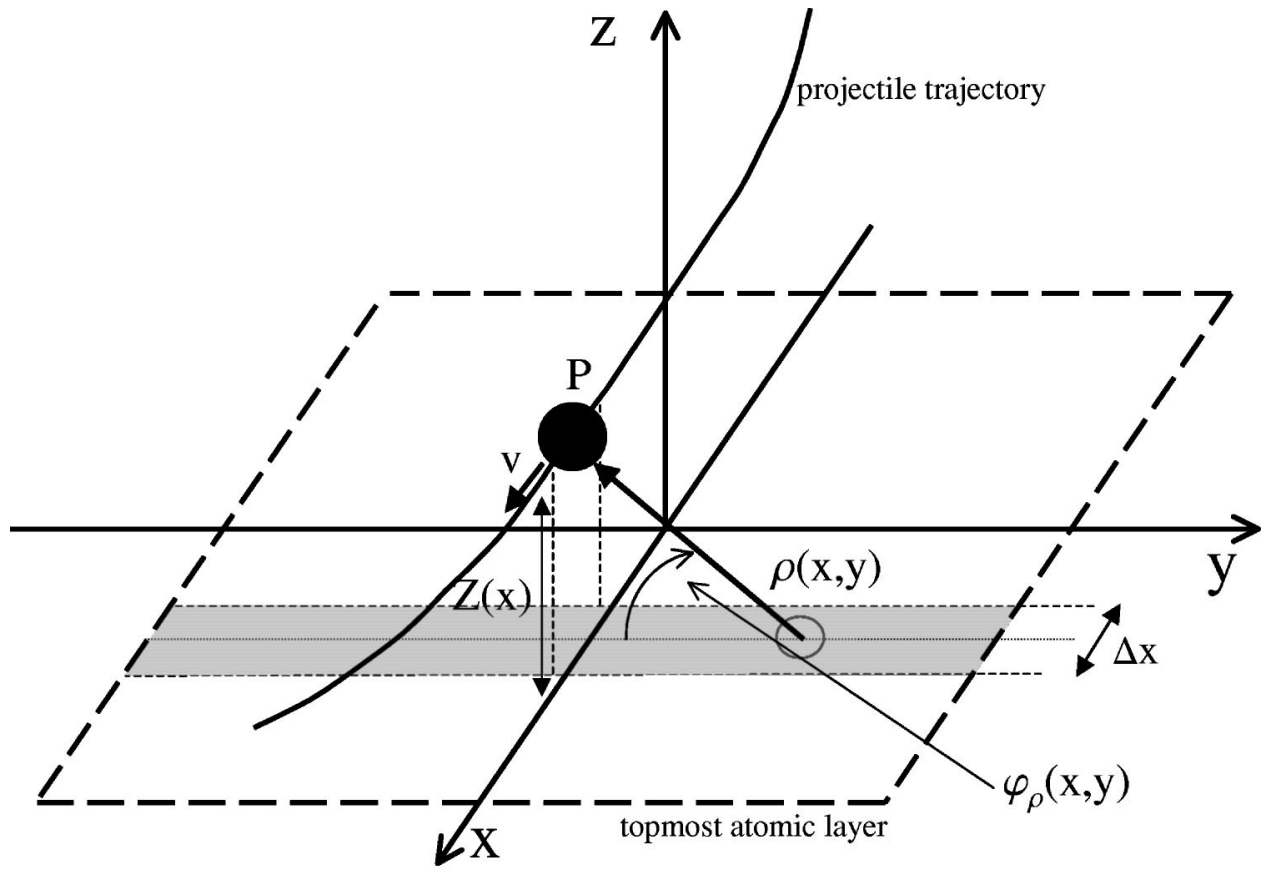

FIG. 1. Schematic picture of the coordinate system. which is measured in vacuum. Due to the large mass $M_{P}$ of the projectile, the description of its motion in terms of a classical trajectory is a reasonable approximation. We use a frame of reference fixed to the position of the first atomic layer, with the projectile trajectory contained in the $x-z$ plane, and the surface in the $x-y$ plane (see Fig. 1). As a consequence of the symmetry of the problem, it is convenient to decompose the projectile velocity into two components: a two-dimensional vector parallel to the surface $\vec{v}_{s}$, and a component perpendicular to the surface $v_{z}$. The initial impact velocity is $\vec{v}_{i}=\left(\vec{v}_{i s}, v_{i z}\right)=\left(v_{i} \cos \theta_{i}, 0,-v_{i} \sin \theta_{i}\right)$, with $\theta_{i}$ the angle of incidence. Atomic units are used unless otherwise stated.

In grazing collisions the ion trajectory can be divided in differential portions situated at different distances $Z(X)$ from the surface. In every portion, the projectile can be supposed to be moving parallel to the surface with velocity $\vec{v}_{s}$. Since target electrons are localized around $\mathrm{F}^{-}$ions, only electrons bound to target ions situated at the first atomic plane contribute effectively to the ionization process. The ionization probability per unit surface area, $d^{2} P_{i}\left(\vec{k}_{f}\right) / d x d y$, for the transition from the initial state $i$ to the final state with momentum $\vec{k}_{f}$, is approximated by

$$
d^{2} P_{i}\left(\vec{k}_{f}\right) / d x d y=\delta_{S} P_{i \vec{k}_{f}}^{(a t)}(\vec{\rho}(x, y)),
$$

where $P_{i \vec{k}_{f}}^{(a t)}(\vec{\rho})$ is the probability of atomic ionization (see the following section). It depends on the impact parameter $\vec{\rho}(x, y)$, where

$$
\rho(x, y)=\sqrt{y^{2}+Z^{2}(x)}, \quad \varphi_{\rho}(x, y)=\arctan \left(\frac{Z(x)}{-y}\right)
$$

are the modulus and the azimuthal angle, respectively. $\delta_{S}$ is the surface atomic density, which is considered to be constant. From Eq. (1) we can derive the ionization probability per unit path length, given by

$$
\frac{d P_{i}}{d x}=\int d \vec{k}_{f} \int_{-\infty}^{\infty} d y \frac{d^{2} P_{i}\left(\vec{k}_{f}\right)}{d x d y}
$$

and the energy loss per unit path length or stopping power,

$$
\frac{d E_{i}}{d x}=\int d \vec{k}_{f} \int_{-\infty}^{\infty} d y \frac{d^{2} P_{i}\left(\vec{k}_{f}\right)}{d x d y}\left(\frac{k_{f}^{2}}{2}-\varepsilon_{i}\right),
$$

as well as the track potential.

When the projectile reaches the position $\vec{R}=(X, 0, Z(X))$ on the classical trajectory, the contribution to the track potential of electronic transitions $i \rightarrow \vec{k}_{f}$ induced by the projectile can be defined as $V_{i \vec{k}_{f}}^{(t r a c k)}(\vec{R}, \vec{r})$, with $\vec{r}$ the position where the track potential is evaluated. Since the surface charge left by the target ionization is proportional to the ionization probability, the partial track potential can be derived from $d^{2} P_{i}\left(\vec{k}_{f}\right) / d x^{\prime} d y^{\prime}$ as

$$
V_{i \overrightarrow{k_{f}}}^{(\text {track })}(\vec{R}, \vec{r})=\int_{-\infty}^{X} d x^{\prime} \int_{-\infty}^{+\infty} d y^{\prime} \frac{d^{2} P_{i}\left(\vec{k}_{f}\right)}{d x^{\prime} d y^{\prime}} \frac{1}{\left|\vec{r}-\vec{r}^{\prime}\right|}
$$

The vector $\vec{r}^{\prime}=\left(x^{\prime}, y^{\prime}, 0\right)$ indicates the position of the differential surface area considered, where the $\mathrm{F}^{-}$ion is located. The integration along the $x$ axis includes all the sites behind the projectile and the $y$-axis integration formally includes all the surfaces. In practice, as the impact-parameter dependence of the ionization probability decays exponentially at large distances, convergency in the integrals is quickly achieved. 
The total track potential produced by ionization from the initial state $i$ is obtained by integrating $V_{i k_{f}}^{(t r a c k)}$ over all final electronic states $\vec{k}_{f}$, i.e.,

$$
V_{i}^{(t r a c k)}(\vec{R}, \vec{r})=\int d \vec{k}_{f} V_{i \vec{k}_{f}}^{(t r a c k)}(\vec{R}, \vec{r}) .
$$

Using symmetry arguments it is observed that the total atomic ionization probability $P_{i}^{(a t)}(\vec{\rho})=\int d \vec{k}_{f} P_{i \vec{k}_{f}}^{(a t)}(\vec{\rho})$ is independent of the orientation of $\vec{\rho}$, i.e., it does not depend on the azimuthal angle $\varphi_{\rho}$. Therefore, it is convenient to change the variable $y^{\prime}$ by $\rho$ in the integral given by Eq. (5), and the track potential reads

$$
V_{i}^{(t r a c k)}(\vec{R}, \vec{r})=\delta_{S} \int_{-\infty}^{X} d x^{\prime} \int_{\left|Z\left(x^{\prime}\right)\right|}^{+\infty} d \rho \rho P_{i}^{(a t)}(\rho) \frac{\mathcal{V}\left(\vec{r}, \rho, x^{\prime}\right)}{d\left(\rho, x^{\prime}\right)},
$$

where $d\left(\rho, x^{\prime}\right)=\left[\rho^{2}-Z\left(x^{\prime}\right)^{2}\right]^{1 / 2}$, and

$$
\mathcal{V}\left(\vec{r}, \rho, x^{\prime}\right)=\frac{1}{\left|\vec{r}-\vec{r}_{+}^{\prime}\right|}+\frac{1}{\left|\vec{r}-\vec{r}_{-}^{\prime}\right|}
$$

represents the Coulomb potential originated by unit charges placed at $\vec{r}_{+}^{\prime}$ and $\vec{r}_{-}^{\prime}$ surface positions, $\vec{r}_{ \pm}^{\prime}=\left[x^{\prime}\right.$, $\left.\pm d\left(\rho, x^{\prime}\right), 0\right]$ being symmetric positions with respect to the scattering plane $(\hat{x}, \hat{z})$. Exchanging the order of integration and replacing the variable $x^{\prime}$ by $z^{\prime}=Z\left(x^{\prime}\right)$, the potential $V_{i}^{(\text {track })}$ reads

$$
V_{i}^{(t r a c k)}(\vec{R}, \vec{r})=\delta_{S} v_{i s} \int_{z_{j}(X)}^{+\infty} d \rho \rho P_{i}^{(a t)}(\rho) \beta_{j}(X, \rho, \vec{r}) .
$$

If $j=i n($ out $)$ indicates that the incident ion is located on the incoming (outgoing) path, with $X<0(X>0)$, then the parameter $z_{j}$ reads

$$
z_{j}(X)= \begin{cases}Z(X), & j=\text { in } \\ Z_{0}, & j=\text { out },\end{cases}
$$

with $Z_{0}=Z(0)$ the distance of closest approach to the surface $\left(Z_{0}>0\right)$. In Eq. (9) the factors $\beta_{\text {in,out }}$ are defined as

$$
\beta_{i n}(X, \rho, \vec{r})=\int_{Z(X)}^{\rho} d z^{\prime} \frac{\mathcal{V}\left(\vec{r}, \rho, x_{i n}^{\prime}\right)}{\left|v_{z}\left(z^{\prime}\right)\right|\left(\rho^{2}-z^{\prime 2}\right)^{1 / 2}}
$$

and

$$
\begin{aligned}
\beta_{\text {out }}(X, \rho, \vec{r})= & \beta_{\text {in }}(0, \rho, \vec{r}) \\
& +\int_{Z_{0}}^{\rho} d z^{\prime} \frac{\mathcal{V}\left(\vec{r}, \rho, x_{\text {out }}^{\prime}\right) \Theta\left[z_{l}(X, \rho)-z^{\prime}\right]}{\left|v_{z}\left(z^{\prime}\right)\right|\left(\rho^{2}-z^{\prime 2}\right)^{1 / 2}},
\end{aligned}
$$

where $z_{l}(X, \rho)=\min [Z(X), \rho], \Theta$ is the unitary Heaviside function, and $v_{z}\left(z^{\prime}\right)$ is the component perpendicular to the surface of the projectile velocity along the classical path. The variable $x_{\text {in (out })}^{\prime}=-(+)\left|Z^{-1}\left(z^{\prime}\right)\right|$ denotes the $x$ position on the incoming (outgoing) projectile trajectory corresponding to the distance $z^{\prime}$ to the surface. The track electric field can be obtained from the gradient of the track potential. The parallel distance traveled by the ion in grazing incidence conditions is typically a hundred times larger than the perpendicular distance. For example, a change in perpendicular distance of 1.3 a.u. for $100-\mathrm{keV}$ protons $\left(v=2\right.$ a.u.) at $\theta_{i}$ $=0.7 \mathrm{deg}$ angle of incidence corresponds to about 100 a.u. in parallel distance and takes 50 a.u. of time ( $1 \mathrm{fm})$. This time is expected to be short compared to the characteristic time for hole recombination (a few picoseconds).

\section{B. Ionization cross section}

In the present work, we employ the CDW-EIS approximation to evaluate the atomic probabilities $P_{i \vec{k}_{f}}^{(a t)}(\vec{\rho})$. Assuming that the nonionized atomic electrons remain "frozen" during the collision, the problem is reduced to one-activeelectron system, and the $T$-matrix element reads

$$
T_{i \vec{k}_{f}}^{C D W-E I S}=\left\langle\chi_{f}^{C D W}\left|W_{f}^{\dagger}\right| \chi_{i}^{E}\right\rangle,
$$

where $\chi_{f}^{C D W}$ is the final CDW wave function, $\chi_{i}^{E}$ is the eikonal wave function, and $W_{f}$ is the final perturbative potential. In the CDW-EIS approximation the $T$-matrix element has a closed expression [13], and the atomic probability can be derived from Eq. (13) by using the well-known eikonal transformation $P_{i \vec{k}_{f}}^{(a t)}(\vec{\rho})=\left|A_{i \vec{k}_{f}}^{C D W-E I S}(\vec{\rho})\right|^{2}[14]$, where

$$
A_{i \vec{k}_{f}}^{C D W-E I S}(\vec{\rho})=\frac{2 \pi}{v_{s}} \int d \vec{\eta} T_{i \vec{k}_{f}}^{C D W-E I S} \exp (i \vec{\eta} \vec{\rho})
$$

is the CDW-EIS transition amplitude, and $\vec{\eta}$ is the component of the transferred momentum perpendicular to $\vec{v}_{s}$.

In the bulk the ionization cross section and stopping cross section are straightforwardly obtained from the abovementioned atomic probabilities by integration over the whole impact parameter range:

$$
\sigma_{i}=2 \pi \int_{0}^{\infty} d \rho \rho P_{i}^{(a t)}(\rho)
$$

and

$$
S_{i}=2 \pi \int_{0}^{\infty} d \rho \rho P_{i}^{(a t)}(\rho)\left(\frac{k_{f}^{2}}{2}-\varepsilon_{i}\right) .
$$

\section{RESULTS AND DISCUSSION}

We present results for $100-\mathrm{keV}$ proton impact on $\mathrm{LiF}(100)$ surfaces at grazing angle of incidence. In this energy range the dominant channel is $\mathrm{F}^{-}$ionization. More precisely, $2 s$ and $2 p$ electrons are considered. In first approximation they are described by Hartree-Fock orbitals for negative ions [15]. The contribution of $\mathrm{Li}^{+} 1 s$ electron ionization has been in- 


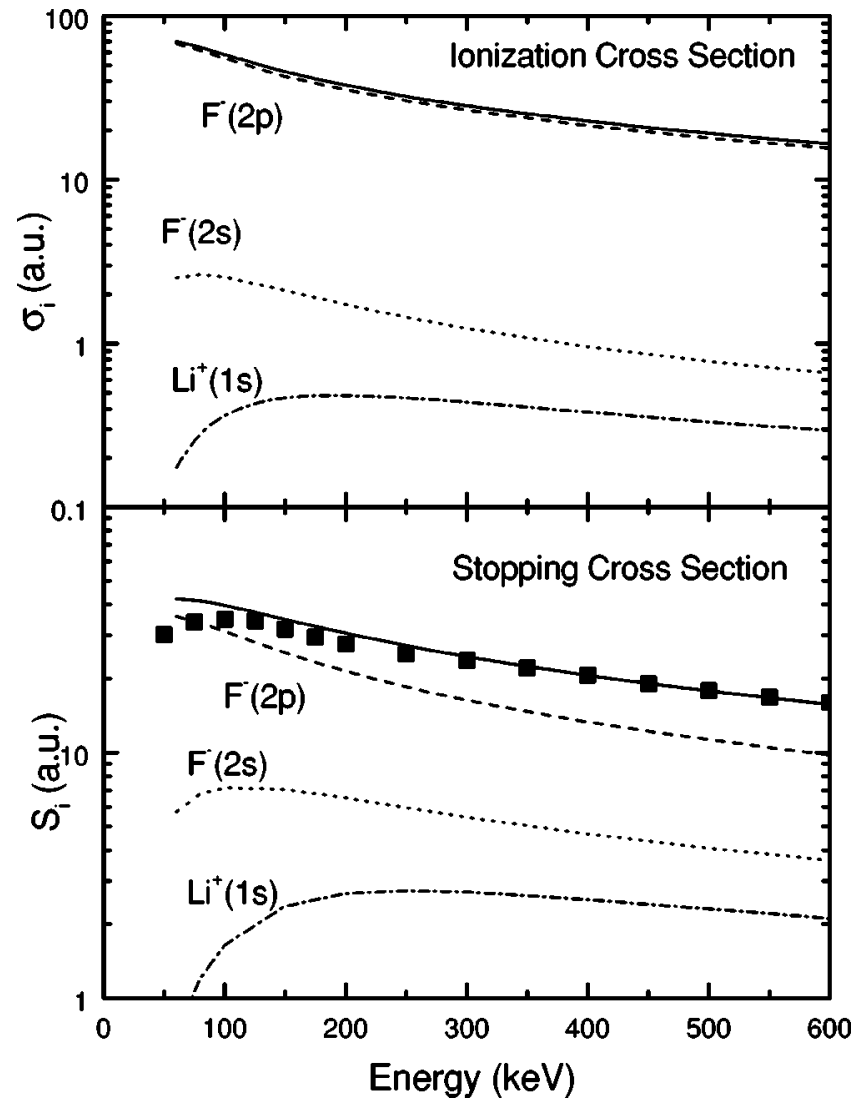

FIG. 2. Ionization cross section and stopping cross section as a function of projectile energy for protons on bulk LiF. The measured stopping cross section data $(\boldsymbol{\square})$ are taken from the work of Bader et al. [17]. The contributions from $\mathrm{Li}^{+}(1 s), \mathrm{F}^{-}(2 s)$, and $\mathrm{F}^{-}(2 p)$ are shown separately.

cluded only to compare with measured stopping cross section data at high energies (see Fig. 2). No effects due to the energy gap of the crystal are considered, as they are not expected to be significant at these high energies where the mean excitation energy is larger than the energy gap. This means that the projectile charge is the perturbation that produces the violent ionization of the target electrons which do not experience effects due to the band structure of the crystal [16]. The track potential calculation involves a fivedimensional integration that we evaluate numerically with a relative error less than $5 \%$. The trajectory integration is done interpolating approximately 20 pivots.

\section{A. Track potential}

As our model for the track potential is based on an evaluation of the surface charge density produced by ionization of the target $\mathrm{F}^{-}$ions by the fast projectile, we study first the ionization cross section and stopping cross section of bulk $\mathrm{LiF}$ for protons in the energy range 50-600 keV. In Fig. 2 we show the energy dependence of both the stopping cross section and ionization cross section of $\mathrm{F}^{-}$by $\mathrm{H}^{+}$impact. These are given by Eqs. (15) and (16). We include LiF stopping cross section data measured by Bader et al. [17]. The overall agreement is rather good. The ionization cross section $(\sigma)$

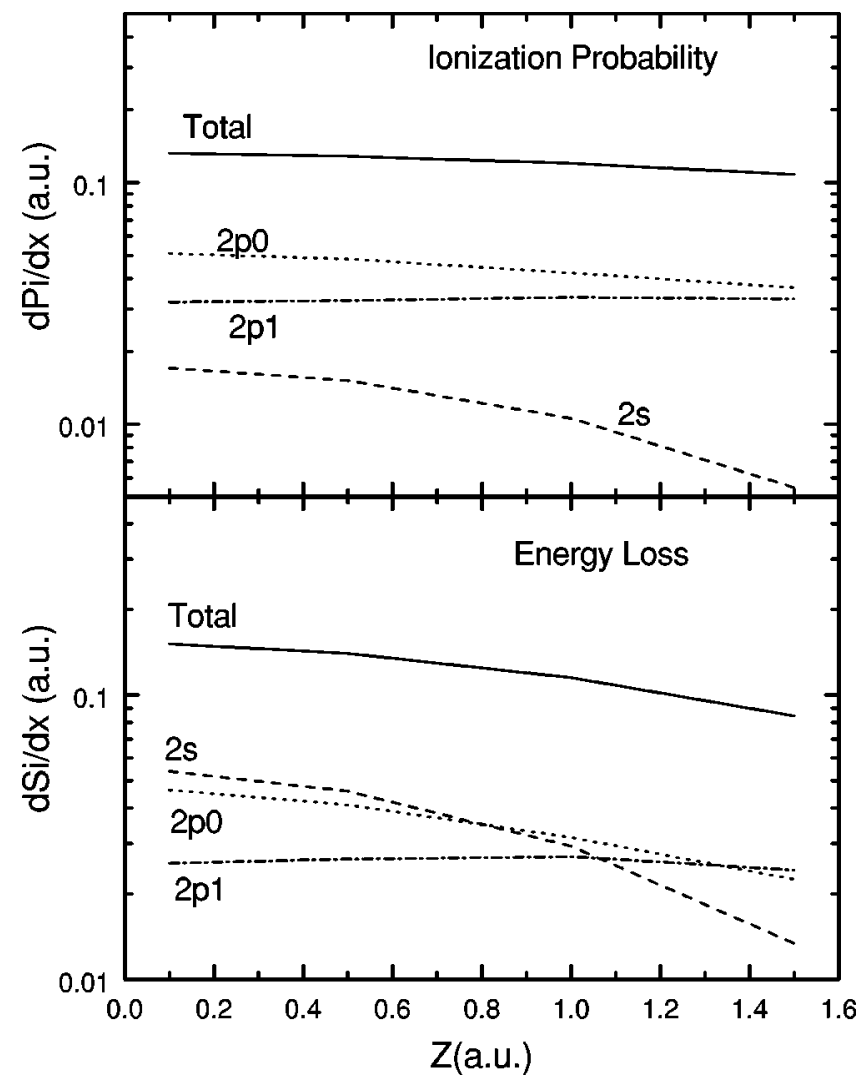

FIG. 3. Ionization probability per unit path length and stopping power as a function of perpendicular distance to the surface for $100-\mathrm{keV}$ protons on a $\mathrm{LiF}(100)$ surface. The contributions from $\mathrm{F}^{-}(2 s), \mathrm{F}^{-}(2 p 0)$, and $\mathrm{F}^{-}(2 p 1)$ are shown separately.

values in the energy range $50-600 \mathrm{keV}$ correspond to ionization probabilities per unit path length $\left(d P / d x=N_{a t} \sigma\right.$, with $N_{a t}=0.00455$ a.u.) of around $0.3-0.1$ a.u. At $100 \mathrm{keV}$ the ionization probability per unit path length is approximately 0.25 a.u. The stopping cross-section values in this energy range $(S)$ correspond to stopping powers $(d E / d x$ $=N_{a t} S$ ) of about 0.2 a.u., or equivalently $10 \mathrm{eV} / \AA$. The ratio of the stopping cross section to the ionization cross section gives us an idea about the mean excitation energy. It is about 1 a.u. in this energy range and, therefore, confirms the approximation of neglecting the energy gap. At $100 \mathrm{keV}$ the dominant contribution to the ionization probability is due to the $2 p$ electrons of $\mathrm{F}^{-}$. However, the stopping cross section has significant contributions from both $2 s$ and $2 p$ electrons of $\mathrm{F}^{-}$, while the $\mathrm{Li}^{+}(1 s)$ contribution is negligible.

Next we study the ion-surface collision situation. In Fig. 3 we show the ionization probability per unit path length and stopping power for $100-\mathrm{keV}$ protons on $\mathrm{LiF}(100)$ as a function of the perpendicular distance to the surface, as given by Eqs. (3) and (4). The ionization probabilities per unit path length vary between 0.12 a.u. close to the surface $(z$ $=0.1$ a.u.) and 0.1 a.u. at $z=1.5$ a.u. These means that close to the turning point of the trajectory, the protons experience a rather intense track potential. The stopping power values vary from 0.15 to 0.08 a.u. in the same perpendicular distance range, i.e., a few $\mathrm{eV} / \AA$. These values are of the same order of magnitude as those calculated using a local plasma 


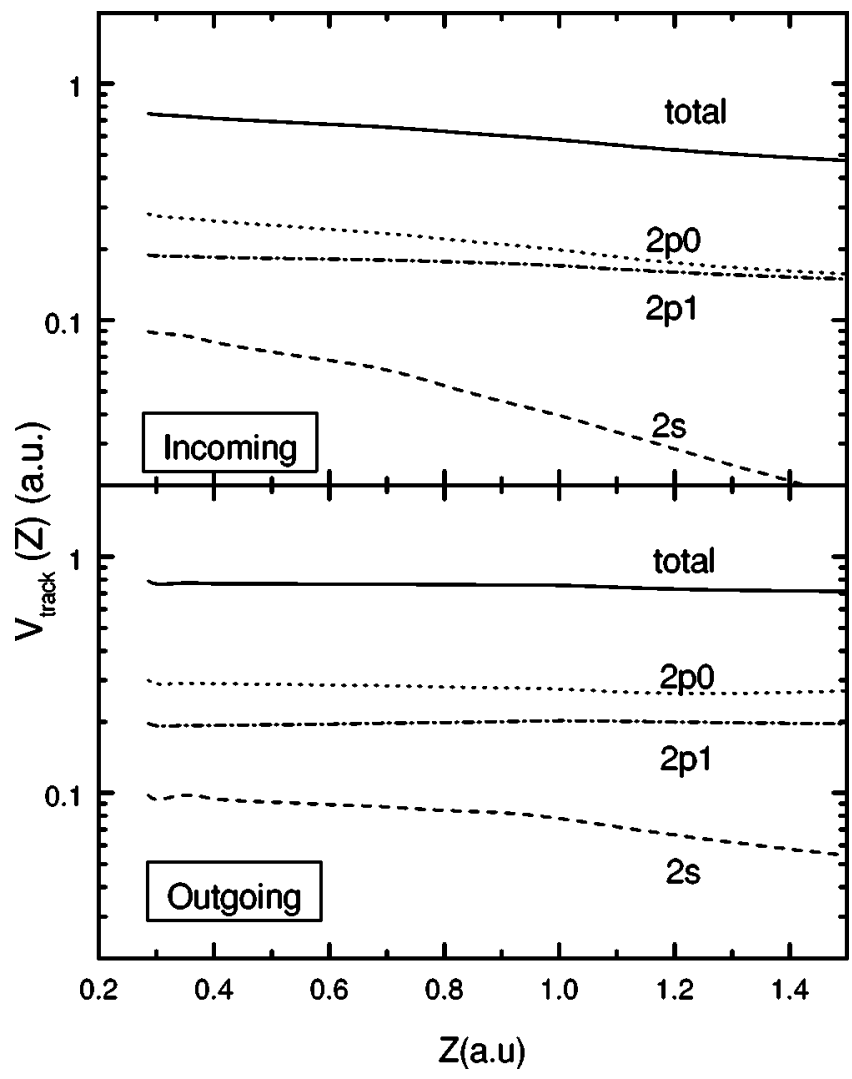

FIG. 4. Track potential evaluated at the proton position as a function of the perpendicular distance to the surface for both incoming and outgoing projectiles of $100 \mathrm{keV}$ under $0.7^{\circ}$ angle of incidence. The contributions from $\mathrm{F}^{-}(2 s), \mathrm{F}^{-}(2 p 0)$, and $\mathrm{F}^{-}(2 p 1)$ are shown separately.

approximation $[18,19]$. At higher energies, surface-plasmon excitation may be an important channel for the energy loss not taken into account by our model, particularly at large distances from the surface. Therefore, it is not able to quantitatively explain higher energy stopping power and electron emission data like those of Kimura et al. [20] for $500-\mathrm{keV}$ protons.

In Fig. 4 we show the perpendicular distance dependence of the track potential for $100-\mathrm{keV}$ protons under $\theta_{i}=0.7 \mathrm{deg}$ angle of incidence on a $\mathrm{LiF}(100)$ surface for both incoming and outgoing ions, i.e., before and after being reflected at the turning point of the trajectory. The track potential is evaluated at the proton position. Close to the surface $(z$ $=0.3$ a.u.), the value of the track potential is about 0.75 a.u. (around $20 \mathrm{eV}$ ). Schiwietz et al. have estimated track potential values from the shift in Auger line positions in the bulk of insulators ionized by fast heavy ions [5], which are of the order of $50 \mathrm{eV}$. The perpendicular distance dependence is rather smooth, as can be seen in the figure. At 1.5 a.u. from the surface, the track potential has decreased $50 \%$ for incoming ions and only $10 \%$ for outgoing ions. This means that it plays a significant role in the electron emission process. We are not only interested in evaluating the track potential [Eqs. (5) and (7)] but also the electric field. In Fig. 5 we show the parallel $(x)$ component of the track electric field as a function of the distance to the surface for the same conditions as in

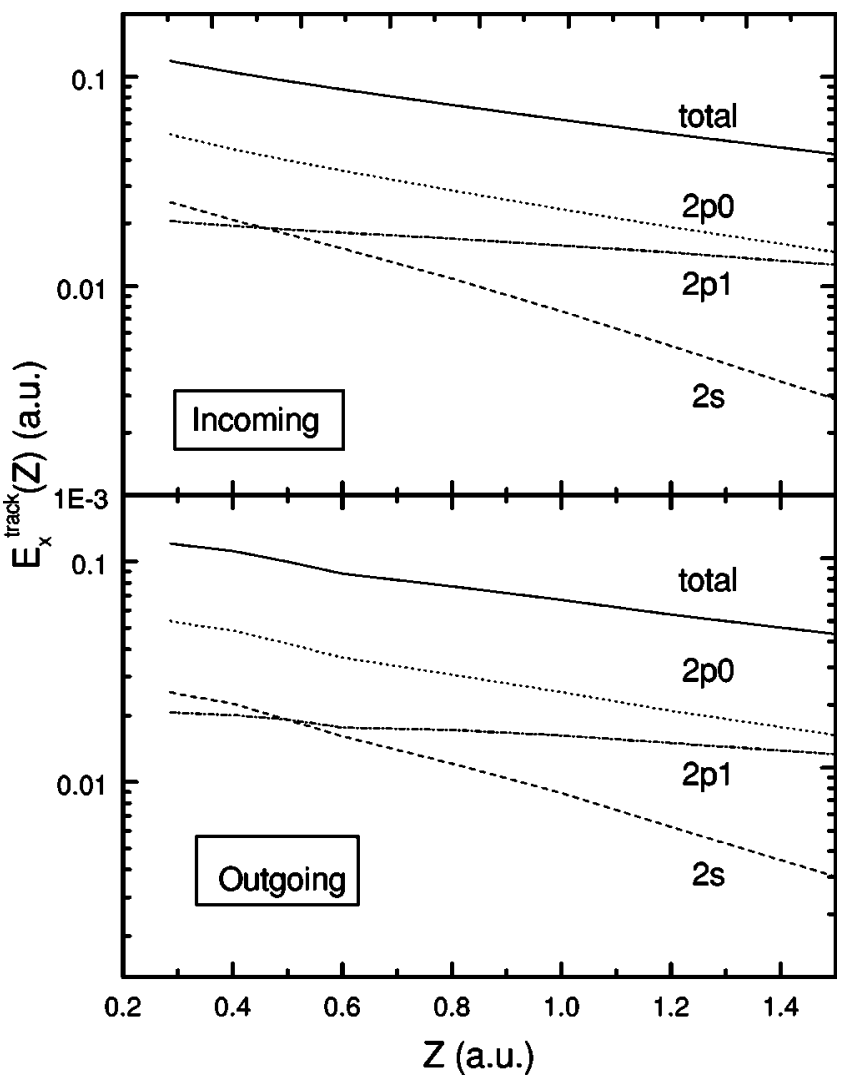

FIG. 5. Parallel track electric-field components as a function of the perpendicular distance to the surface (same conditions as in Fig. 4).

Fig. 4. The values of the parallel component of the electric field are of the order of 0.1 a.u. close to the top most layer ( $z=0.3$ a.u.) and half of it at 1.5 a.u., where most of the ionization processes takes place in grazing incidence conditions. In Fig. 6 we show the perpendicular $(z)$ component of the track electric field as a function of the distance to the surface for the same conditions as in Fig. 5. The values of the parallel component of the electric field are smaller than the parallel component (approximately one-half).

There are effects such as screening by low-energy electrons or polarization of the neighbor anions that may reduce the strength of the track potential and, consequently, the track field as well. Let us first consider the approximation of neglecting screening by low-energy electrons. The electrons emitted from a given ion travel preferentially in the forward direction, that is, away from the track. As the closest site of a previous ionization processes is 7.6 a.u. behind, it is possible to assume that the majority of the electrons will escape from the positively charged surface. In fact, high electron emission yields are measured [21]. Concerning the effects due to polarization of the neighboring $\mathrm{F}^{-}$anions, it is important to notice that it is the electronic polarizability which is relevant in the time scale of the projectile motion, and not the ionic polarizability. Since the optical dielectric constant of $\mathrm{LiF}$ is $\varepsilon=1.96$, an upper bound for the reduction of the track potential at the surface due to this effect is a factor of 2 (between $1 / \varepsilon$ (bulk) and $1-[(\varepsilon-1) /(\varepsilon+1)]($ surface $)$ ). 


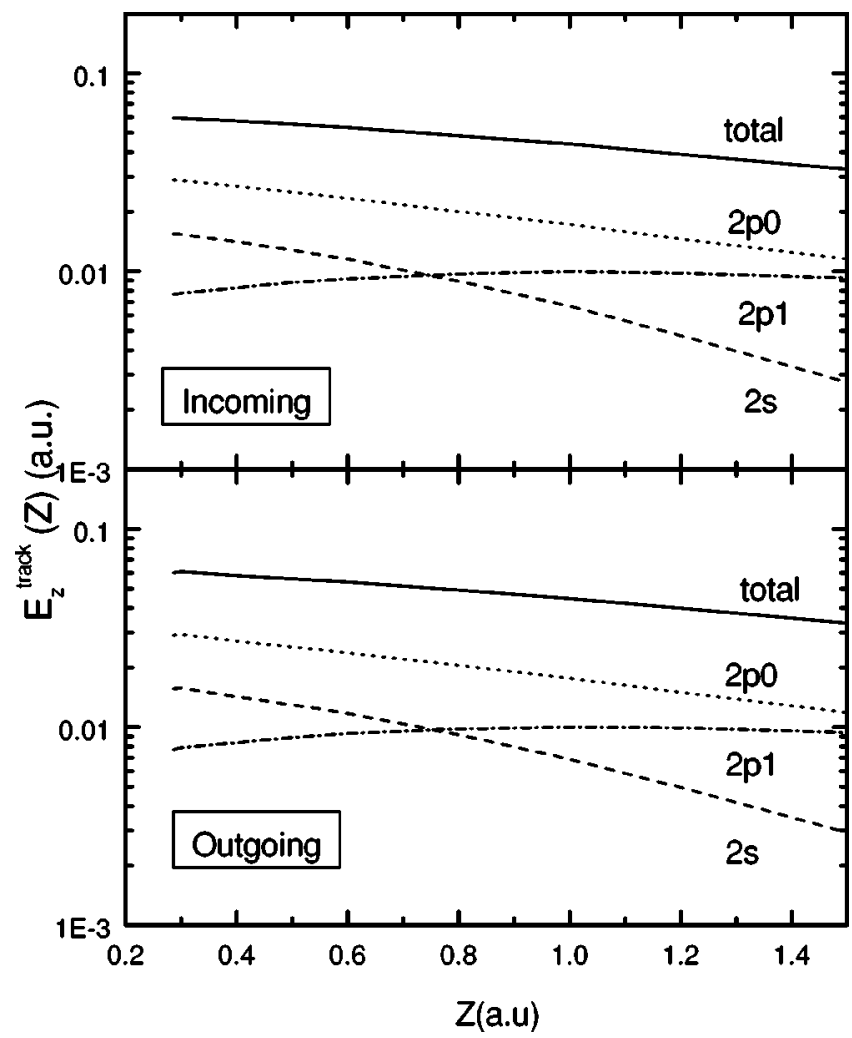

FIG. 6. Perpendicular track electric-field components as a function of the perpendicular distance to the surface (same conditions as in Fig. 4).

\section{B. Convoy shift}

The value of the parallel component of the track electric field can be used to estimate the change in the pure Coulomb ionization threshold at zero energy due to a constant electric field (a Stark-type model). In Fig. 7 we plot the onedimensional potential in the $x$ direction,

$$
V\left(x_{p}\right)=-\frac{q}{\left|x_{p}\right|}+E_{x}^{\text {track }} x_{p},
$$

$x_{p}$ is the relative electron-projectile coordinate along the direction of motion, while $E_{x}^{\text {track }}$ is the parallel component of the electric field generated by the track potential at the projectile site. Its value depends on the projectile distance from the surface $Z(X)$ going from a maximum ( $E_{x}^{\text {track }}$ $=0.12$ a.u.) at the distance of closest approach to zero asymptotically. At a distance of 1.5 a.u. from the surface, its value is $E_{x}^{\text {track }}=0.047$ a.u. A reduction $\left(\Delta V=-2 \sqrt{E_{x}^{\text {track }}}\right)$ of the pure Coulomb ionization threshold $(V=0)$ for backward ionization appears. Its maximum value at the turning point of the trajectory is reduced as the ion leaves the surface. At $z=1.5$ a.u., it is about 0.4 a.u. $(10 \mathrm{eV})$.

However, the convoy electron (the one that has a velocity close to the ion velocity, i.e., $\vec{k}_{f} \approx \vec{v}$ ) suffers the simultaneous attraction by the surface track and the repulsion from the electrons originated in $\mathrm{F}^{-}$ionizations. Some of these electrons will move away from the surface and projectile in such a way that their interaction with the convoy electron will

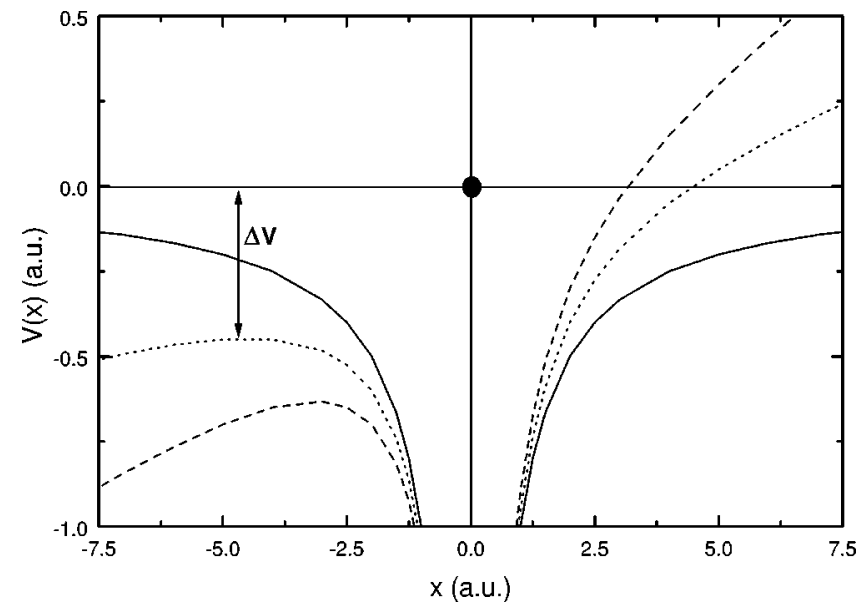

FIG. 7. Effective one-dimensional potential (Coulomb plus constant electric field). The solid line is the bare Coulomb potential $\left(E_{x}^{\text {track }}=0\right)$, the dotted line corresponds to $E_{x}^{\text {track }}=0.1$ a.u., and the dashed line to $E_{x}^{\text {track }}=0.05$ a.u.. A reduction of the ionization threshold $\Delta V=-2 \sqrt{E_{x}^{\text {track }}}$ appears for backward ionization. See the text for details.

influence the motion of the latter along the forward direction. On the one hand, approximately half of these electrons will travel inside the crystal with large mean free paths. On the other hand, the repulsive interaction between pairs of electrons at a given distance $V_{e}$ is shared between the two of them; the convoy electron will acquire an increment in velocity, $\Delta v_{e}$, given by $\frac{1}{2}\left(\Delta v_{e}\right)^{2}=\frac{1}{2} V_{e}$. On the contrary, for the interaction between the convoy electron and the positive ion charge of the track, due to the large ion mass, all the potential energy is given to the electron, which is decelerated. Therefore, the attractive interaction between the convoy electron and the surface track will be only slightly reduced by these electron clouds of ionized electrons.

The presence of an electric field as that shown in Fig. 7 lowers the ionization threshold of the projectile and makes it anisotropic. When the projectile-surface collision evolves along the classical trajectory, electron states form an outgoing wave packet centered on the ion. The evolution of each orbital of this wave packet makes those electrons with energies above the threshold in the projectile frame to move away. The anisotropy in the threshold enhances the population of continuum states moving backwards in the projectile frame and depletes the spectrum of forward electrons in this frame. Although it is difficult to directly relate the magnitude of the shift in the maximum of the electron distribution with the lowering of the ionization threshold in the backward direction, they will be of the same order of magnitude [22]. This means that for $100-\mathrm{keV}$ protons, a reasonable upper bound for the shift is $10 \mathrm{eV}$. This value would decrease for lower projectile energies due to the reduction of the strength of the track. In this energy range close to its maximum, the ionization cross section is higher at lower energies, when all impact parameters are available. However, in grazing incidence there is a reduction of small-impact-parameter collisions where ionization is more efficient. Consequently, the reduction of the strength of the track potential at lower energies would appear as an effect due to a difference in the ion 
trajectory, which reflects larger distances of closest approach. A more refined calculation would require to follow in detail the evolution of the electron charge centered on the projectile. Therefore, we can say that for low-energy electrons in the frame of reference of the projectile, the effect of the track potential is to favor a shift to backward velocities of the convoy electrons that will be emitted with energies below the unperturbed cusp energy typical of the convoy electrons. They will appear in the electron spectra as a shoulder shifted to low energies with respect to the pure cusp, which is markedly reduced.

\section{CONCLUSION}

The surface track potential plays a significant role in electron emission from insulating surfaces by impact with fast ions under grazing incidence conditions. A simple model based on a surface charge density calculated from ionization of $\mathrm{F}^{-}$sites allows us to estimate the strength of the track potential and electric field. We find that for $100-\mathrm{keV}$ protons on $\mathrm{LiF}(100)$ surfaces, the track potential may be of the order of $10 \mathrm{eV}$ close to the surface $(z=1.6$ a.u.), and the parallel component of the corresponding electric field is about
$2 \mathrm{eV} / \AA$. This track potential could produce shifts to lower energies of the convoy electron peak position of a few $\mathrm{eV}$, as measured in the experiments. A first qualitative estimate of this shift is based on a Stark-type calculation of the reduction of the Coulomb ionization threshold in constant field approximation. Other effects, such as screening by low-energy electrons or polarization of the surrounding anions, might reduce the strength of the track potential. Therefore, more experiments done under different conditions (changing targets, projectile energy, geometry, ...) as well as more specific calculations [23], will help in understanding the problem.

\section{ACKNOWLEDGMENTS}

We are grateful to K. Kimura, E. A. Sanchez, O. Grizzi, and M. L. Martiarena for helpful discussions. A.A. acknowledges support by the DIPC, UPV/EHU, Eusko Jaurlaritza, and the Spanish MCYT (Contract No. BFM2001-0076). V.H. would like to thank the DIPC for financial support. M.S.G. and J.M. were partially supported by the ANPCyT (Project Nos. PICT03-03579 and PICT03-06249) and the UBACyT (Project No. 01-X044).
[1] L. Hägg, C.O. Reinhold, and J. Burgdörfer, Phys. Rev. A 55, 2097 (1997).

[2] J.J. Ducrée, F. Casali, and U. Thumm, Phys. Rev. A 57, 338 (1998).

[3] A.G. Borisov, V. Sidis, and H. Winter, Phys. Rev. Lett. 77, 1893 (1996).

[4] P.M. Echenique, F. Flores, and R.H. Ritchie, Semicond. Sci. Technol. 43, 230 (1990).

[5] G. Schiwietz et al., Phys. Rev. Lett. 69, 628 (1992).

[6] G. Xiao et al., Phys. Rev. Lett. 79, 1821 (1997).

[7] G.R. Gomez, O. Grizzi, E.A. Sanchez, and V.H. Ponce, Phys. Rev. B 58, 7403 (1998).

[8] K. Wien, Ch. Koch, and N. van Tan, Nucl. Instrum. Methods Phys. Res. B 100, 322 (1995).

[9] K. Kimura, Nucl. Instrum. Methods Phys. Res. B 193, 661 (2002)

[10] M.S. Gravielle, Phys. Rev. A 62, 062903 (2000).

[11] V.G. Moliere, Z. Naturforsch. 133, 2 (1947).

[12] P.D. Fainstein, V.H. Ponce, and R.D. Rivarola, J. Phys. B 24, 3091 (1991).

[13] P.D. Fainstein, V.H. Ponce, and R.D. Rivarola, J. Phys. B 22,
1207 (1989).

[14] M.R.C. McDowell and J.P. Coleman, Introduction to the Theory of Ion-Atom Collisions (North-Holland, Amsterdam, 1970).

[15] E. Clementti and C. Roetti, At. Data Nucl. Data Tables 14, 177 (1974).

[16] J.I. Juaristi et al., Phys. Rev. Lett. 84, 2124 (2000).

[17] M. Bader, R.E. Pixley, F.S. Mozer, and W. Whaling, Phys. Rev. 103, 32 (1956).

[18] A. Sarasola, V.H. Ponce, and A. Arnau, Nucl. Instrum. Methods Phys. Res. B 203, 104 (2003).

[19] J.E. Miraglia and M.S. Gravielle, Phys. Rev. A 66, 032901 (2002).

[20] K. Kimura, G. Andou, and K. Nakajima, Nucl. Instrum. Methods Phys. Res. B 164/165, 933 (2000).

[21] K. Kimura, G. Andou, and K. Nakajima, Phys. Rev. Lett. 81, 5438 (1998).

[22] E.A. Sanchez, O. Grizzi, M.L. Martiarena, and V.H. Ponce, Phys. Rev. Lett. 71, 801 (1993).

[23] M.S. Gravielle and J.E. Miraglia, Phys. Rev. A 67, 042901 (2003). 